\title{
Qualitätsmanagement in der ambulanten Versorgung: mögliche Rollen der FMH
}

\author{
K. Wyss, J. Schwarz, \\ H. Prytherch, P. Bovier, \\ M. Hersperger, M. Zahorka
}

1 Wagner C, Gulácsi L, Takacs E, Outinen M. The implementation of quality management systems in hospitals: a comparison between three countries. BMC Health Serv Res. 2006;6:50.

2 Geschäftsprüfungskommission des Ständerats. Die Rolle des Bundes bei der Qualitätssicherung nach KVG. Bericht der Parlamentarischen Verwaltungskontrolle (PVK) der Geschäftsprüfungskommission des Ständerates. Bern, September 2007.

Korrespondenz:

PD Dr. med. Kaspar Wyss

Swiss Centre for International Health Swiss Tropical Institute

Socinstrasse 57

CH-4002 Basel

Tel. 0612848140

Fax 0612848103

kaspar.wyss@unibas.ch

\section{Zusammenfassung}

Die Verbesserung der Qualitätssicherung bei der Patientenversorgung verbunden mit zunehmendem Kostendruck nicht nur im stationären Bereich sondern zunehmend auch in der ambulanten ärztlichen Versorgung stellt die FMH vor Herausforderungen. Unter Aufarbeitung und Berücksichtigung der Erfahrungen anderer europäischer Länder zeigt der vorliegende Artikel drei mögliche Szenarien auf, wie sich die FMH zu diesem Thema stellen kann.

Das erste Szenario besteht in einem Fokus auf die traditionelle Rolle selbstregulativer Mechanismen durch Standesorganisationen. Das zweite legt den Schwerpunkt auf die Einführung laufender Qualitätsverbesserungsmassnahmen wie zum Beispiel durch Qualitätszirkel oder Zertifizierungen. Das dritte Szenario stellt die Schaffung und Etablierung neuer Institutionen, die von der FMH und anderen Partnern getragen werden, in den Vordergrund.

\section{Hintergrund}

Seit den 90er Jahren des vergangenen Jahrhunderts sind in vielen Ländern Europas und Nordamerikas die gesetzlichen Rahmenbedingungen für die Einführung qualitätssteigernder und kostendämpfender Massnahmen im Gesundheitswesen mit oder ohne Nachweispflicht geschaffen worden. Im Fokus der Reformbemühungen war zunächst die stationäre Versorgung und dementsprechend wurden die Spitäler zu grösserer Transparenz und Rechenschaft ihrer Tätigkeiten angehalten [1]. In jüngerer Vergangenheit hat die Diskussion jedoch auch den ambulanten Sektor erfasst und in vielen Ländern existieren bereits Modellprojekte in Gruppenpraxen oder Praxisverbänden.

Die Einführung von Qualitätsmanagement (QM) im Gesundheitswesen droht das bisherige Model ärztlicher Selbstregulierung und die Rolle der ärztlichen Standesorganisationen nachhaltig zu verändern. Neue Akteure (z. B. Behörden, Organisationsberatungen und Zertifizierer, Patientenverbände usw.) betreten das Feld und nehmen Einfluss auf die Rahmenbedingungen medizinischer Tätigkeit. Die Diskussion wird ebenfalls in der Schweiz engagiert geführt, und der letztjährige Bericht der parlamentarischen Verwaltungskontrolle (PVK) zeigt, dass der Bund aktiv Stel- lung beziehen muss [2]. Vor diesem Hintergrund stellen sich die Fragen nach der zukünftigen Rolle der Ärzteverbände für die Qualitätssicherung (QS) in der ambulanten Versorgung in der Schweiz, nach der Rolle der ärztlichen Selbstkontrolle in der Zukunft und den Möglichkeiten, die sich für die FMH bieten, um auf die neuen Anforderungen zu reagieren.

Der vorliegende Artikel vermittelt einen Einblick in die Thematik und zeigt Entwicklungstendenzen in verschiedenen Ländern Westeuropas und Nordamerikas auf. Zudem werden mögliche Positionierungen der FMH bei der Einführung von QM-Instrumenten in der ambulanten Versorgung diskutiert.

\section{Qualitätsmanagement (QM) im Gesundheitswesen}

Neben den klassischen Instrumenten der Qualitätssicherung (QS) medizinischen Handelns (z. B. Standardisierung, Aus- Weiterund Fortbildungsordnung, Zertifizierung) sind in den letzten Jahrzehnten zunehmend aus Produktions- und Dienstleistungssektoren transferierte Konzepte zum kundenorientierten Management qualitätsrelevanter Prozesse übernommen worden. Das Qualitätsmanagement (QM) im Gesundheitswesen geht dabei über den QS-Ansatz hinaus, indem es nicht nur die Qualität der medizinischen Leistung, sondern auch alle qualitätsrelevanten Prozesse betrachtet und diese im Lichte der «Kundenzufriedenheit» optimiert. Der Kundenbegriff bezieht sich dabei nicht nur auf den Patienten, sondern auch auf andere interessierte Gruppen wie Krankenkassen, Gesetzgeber und Gesamtgesellschaft.

\section{Akteure des Qualitätsmanagements}

Die Rolle der ärztlichen Berufsverbände bei der Regulierung und Überwachung medizinischer Versorgungsqualität in der ambulanten Versorgung hängt von der Organisation des jeweiligen Gesundheitssystems ab. In Ländern wie Deutschland, den Niederlanden, Dänemark oder den USA bestehen klassische Aufgaben in der Repräsentation der Interessen ihrer Mitglieder und deren Lizenzierung, der Anerkennung fachärztlicher 
Weiter- und Fortbildung sowie in der Verhandlung von Preistarifen mit Finanzierungsinstitutionen, zum Beispiel mit Krankenkassen.

Qualitätssicherung (QS) in der ambulanten medizinischen Versorgung wurde bis vor kurzem weitgehend als die Aufgabe freiwilliger ärztlicher Selbstkontrolle durch die Standesorganisationen gesehen. Wesentliche Instrumente waren und sind hierbei die Lizenzierung von Ärzten und die kontinuierliche medizinische Weiter- und Fortbildung (CME: continuous medical education). Letztere besteht häufig aus Selbststudium sowie der Teilnahme an Kongressen und Fachtagungen.

Je nach Land sind jedoch in den letzten Jahren neue Aufgaben und Funktionen hinzugekommen, die teilweise auch von neu geschaffenen Akteuren wahrgenommen werden. Beispiele sind:

- Förderung der QS sowie Evaluierung der Qualität ärztlicher Dienstleistungen;

- Entwicklung klinischer Standards («clinical practice guidelines») innerhalb von Prinzipien der evidenzbasierten Medizin;

- Qualitätskontrolle und Audits;

- Messung der Produktivität, der Performance und des Outcome medizinischer Dienstleistungen;

- Definition von Standards zum Beispiel durch Evaluation von Dienstleistungen, medizinischen Technologien und Medikamenten;

- Zertifizierung;

- Qualitätsmanagement und «Total Quality Management».

Die neuen Qualitätsanforderungen und die damit einhergehende Dienstleistungen werden zum Teil von bestehenden Organisationen wahrgenommen, zum Teil fördern sie aber auch die Entstehung neuer, unabhängiger Organisationen oder die Zusammenarbeit bestehender Institutionen in ausgegliederten Gruppierungen. In Deutschland wurden zum Beispiel in den vergangenen Jahren beträchtliche Forschungsmittel zur Entwicklung von QM-Instrumenten bereitgestellt, die spezifisch auf die Verwendung im Gesundheitssektor zugeschnitten sind [3]. Aus dem Forschungsprojekt ist eine unabhängige Organisation entstanden - die Kooperation für Transparenz und Qualität (KTQ) -, die Zertifizierungsangebote für Spitäler, Rehabilitationseinrichtungen und neuerdings auch ambulante Praxen offeriert. Andererseits hat die Kassenärztlichen Bundesvereinigung (KBV) Deutschlands ein eigenes Zertifizierungsangebot für den ambulanten Bereich entwickelt, «Qualität und Entwicklung in Praxen»(QEP). Beispiele in der Schweiz sind der Verein Outcome oder die Stif- tung EQUAM. Der Verein Outcome in Zürich ist aus der Zusammenarbeit kantonaler Gesundheitsverwaltungen mit Spitälern und Krankenkassen entstanden und führt regelmässig Evaluierungen der Qualitätsstandards in den Mitgliederspitälern durch. Die Resultate werden den Interessenten zurückgemeldet. Über einen Benchmarkingprozess soll dies zur Qualitätsverbesserung der stationären Versorgung führen. EQUAM dagegen ist als Stiftung organisiert und gewährt ihren Mitgliedern aus dem ambulanten Sektor Zugang zu einem Zertifizierungsverfahren.

Ebenso haben in Ländern wie Frankreich, den Niederlanden oder Grossbritannien die Bestrebungen zur Stärkung von Qualität in der ärztlichen Versorgung durch QM-Systeme neue Institutionen kreiert [4, 5]. Ärzteverbände gehen Koalitionen mit anderen Interessensgruppierungen wie Krankenkassen, Akkreditierungsagenturen oder ökonomischen Evaluierungsagenturen ein, um ihre führende Rolle bei der QS zu bewahren und Expertise bei der Einführung von QM-Systemen zu gewinnen. In Frankreich wurde zum Beispiel die «Haute Autorité de Santé» (HAS) als wissenschaftliche und öffentlich-rechtliche, jedoch unabhängige Institution geschaffen, in die auch die frühere Akkreditierungsagentur «Agence nationale d'accréditation et d'évaluation en santé» (ANAES) integriert wurde. In Dänemark hat der Ärzteverband sein Mandat auf wissenschaftliche Referenzfunktionen ausgedehnt.

Die Evaluierung klinischer Abläufe und die Messung von Ergebnissen medizinischer Behandlung haben ebenfalls zur Diskussion der Frage angeregt, welche medizinischen Prozeduren finanziert oder nicht finanziert werden sollen (Negativ- oder Positivlisten). Hierzu haben verschiedene Länder neue Institution geschaffen: Das «National Institute for Health and Clinical Excellence» in Grossbritannien oder die «Haute Autorité de Santé» in Frankreich.

Speziell in Ländern mit staatlichen Finanzierungsmechanismen haben Ärzteverbände ihre führende Rolle bei der QS zugunsten regulierender Institutionen verloren. Das National Health System (NHS) in Grossbritannien, das ursprünglich lediglich die Finanzierung von Gesundheitsdiensten regelte, hat mittlerweile NHS «trust boards» eingerichtet, die sowohl Aufgaben in der QS übernehmen als auch die Prozesse der medizinischen Leistungserbringung im Sinne eines QM-Systems beeinflussen.

\section{Instrumente und Finanzierung}

Die Wahl der Instrumente, die im QM in Gesundheitssystemen eingesetzt werden, variiert. 
Moderne QM-Systeme sind eher auf die Prävention von Fehlern und die «Zufriedenstellung der Kundenerwartungen» als auf Kontrolle festgelegter Massnahmen ausgerichtet, wobei sich dies nicht ausschliesst. Daher werden in der Regel Methoden zur Selbstevaluierung eingesetzt, die im Bedarfsfall mit Fremdevaluierung (audit) oder kollegialen Diskussionen (peer review) ergänzt werden. Zwar ist eine Zertifizierung für die Einführung eines QM-System nicht generell erforderlich, sie wird jedoch von vielen Interessenten als Dokumentation des Erreichten genutzt. Die Zertifizierung einer Institution gemäss einem bestimmten QM-System erfordert in der Regel die Erfüllung gewisser Anforderungen (z.B. ISO 9001). In der Regel kommen wissenschaftlich validierte Fragebogen, «total quality management»-Schemata, Forderungskataloge, mehr oder weniger offene kollegiale Diskussionen inklusive Erarbeitung von Qualitätsvereinbarungen und andere Instrumente zur Anwendung. Die Konformität mit den Anforderungen wird evaluiert und den Leistungserbringern zurückgemeldet. Dies kann zur Einleitung von Verbesserungsmassnahmen oder gegebenenfalls zur Zertifizierung genutzt werden.

Die Definition von Kennzahlen (Indikatoren) aus den kritischen Bereichen der Leistungserbringung und deren regelmässige Evaluierung sind in QM-Systemen zur Identifikation von Verbesserungspotentialen unerlässlich. Im britischen NHS zum Beispiel wurden Verfahren zur Messung von Performance und Qualität der Leistungserbringer erarbeitet, die zur Leistungsvergütung benutzt werden [6]. Angelehnt an Grundprinzipien von QM-Systemen, schliessen diese auch Aspekte wie Arbeitsabläufe und Zugang zu Gesundheitsdiensten (z. B. Wartezeit, Öffnungszeiten), interdisziplinäre und interinstitutionelle Zusammenarbeit zwischen Ärzten und Pflegepersonal respektive zwischen ambulantem und stationärem Sektor ein.

Üblicherweise werden die QM-Systeme in der ambulanten Versorgung hauptsächlich durch die Gesundheitsdienstleister selbst finanziert. Nur in wenigen Fällen, in denen dem Staat eine tragende Rolle zufällt (insbesondere in Gesundheitssystemen wie in Grossbritannien), werden die Kosten der QS durch die Finanzierer mitgetragen. Allerdings gibt es in einigen Ländern indirekte Nutzen für ambulante Versorger: In Frankreich führt die Zertifizierung von Praxen zu einer Reduktion von Versicherungsprämien, und in Deutschland ist es für zertifizierte Leistungserbringer unter Umständen möglich, bessere Preise mit Krankenkassen zu verhandeln. In Ländern, in denen berufliche Haftung eine starke Position hat, ist der Nutzen in Form von Schutz gegen Klagen durch QM-Systeme sicherlich grösser.

\section{Mögliche Rollen der FMH}

Der FMH bieten sich verschiedene Optionen zur Positionierung in der Frage der Einführung von QM-Systemen in der ambulanten Versorgung. Basierend auf den Erfahrungen anderer Länder wie Grossbritannien, Deutschland oder Frankreich und unter Einbezug der inhaltlichen und politischen Debatte zu QM und QS im Gesundheitswesen, wie zum Beispiel des Berichts der parlamentarischen Verwaltungskontrolle (PVK), [2] lassen sich drei Szenarien skizzieren:

- Fokus auf Qualitätssicherung;

- Fokus auf die Einführung neuer Instrumente zu QS/QM;

- Fokus auf die Schaffung und Etablierung neuer Institutionen/Kooperationen zu QS/QM.

\section{Szenario 1 mit Fokus auf Qualitätssicherung}

Die Fokussierung auf QS der ärztlichen Dienstleistung entspricht einer Konzentration auf das derzeitige Kerngeschäft der FMH. Schwerpunkte sind die ärztliche Weiter- und Fortbildung, Curriculumweiterentwicklung und Förderung auf Evidenz basierender medizinischer Abläufe in der ambulanten Praxis. Eine weitere Möglichkeit wäre, Forschungsaktivitäten - vor allem der angewandten Forschung - in Zusammenarbeit mit Universitäten und Fachhochschulen zu stärken und den Transfer neuer wissenschaftlicher Erkenntnisse $\mathrm{zu}$ ambulanten Versorgern $\mathrm{zu}$ fördern. Entwicklungsmöglichkeiten würden sich ausserdem in der Verbesserung von Weiter- und Fortbildungsaktivitäten im Sinne der CME und deren Umsetzung in die Praxis bieten. Wesentliches Verfahren zur QS wäre dabei die Disseminierung neuer Erkenntnisse und die Förderung der Anwendung in der Praxis. Hierbei käme eine Schlüsselrolle den medizinischen Fachgesellschaften zu. In Anbetracht der derzeitigen Aktivitäten der FMH bietet dieses Szenario den Vorteil, auf den derzeitigen Kompetenzen aufzubauen und sich als Garant für kompetente ärztliche Dienstleistungen zu profilieren.

Dieser Ansatz birgt hingegen das folgende Risiko: Unter dem wachsenden Druck zur Einführung neuer QS-Instrumente, die sich zusätzlich den institutionellen und Prozessdimensionen der Qualität im Hinblick auf ein umfassenderes Qualitätsmanagement annehmen, können andere Akteure auftreten und die Lücke füllen. Beispiele dafür sind der Verein Outcome oder die Stiftung EQUAM. Dies kann für die FMH einen Reeves D, Kontopantelis E, performance programs in family practices in the United Kingdom. N Engl J Med. 2006;355(4):375-84 
Einflussverlust in der Diskussion um regulative Massnahmen bedeuten.

\section{Szenario 2 mit Fokus auf die Einführung neuer Instrumente der QS/des QM}

Basierend auf den Erfahrungen und Diskussionen in anderen dezentralisierten Gesundheitssystemen zeigt das zweite Szenario, dass Ärzteverbände verschiedene Instrumente zur QS im Hinblick auf ein umfassenderes Qualitätsmanagementsystem in der ambulanten Versorgung fördern können. Der Schwerpunkt geht dabei über die üblichen Aspekte der Qualitätssicherung hinaus und betrachtet ebenfalls Aspekte institutioneller und Prozessqualität. Die Beratungsfunktion der FMH bezüglich Methodenkompetenz würde erweitert. Mögliche Instrumente, die für die Mitglieder nutzbar gemacht werden könnten, wären Qualitätszirkel, «peer audits», Selbstevaluierungen bis hin zur Vorbereitung auf Zertifizierungen.

Die FMH könnte dabei die Rolle einer Moderatorin einnehmen und kontinuierliche Qualitätsverbesserungsinitiativen unter ihren Mitgliedern fördern. Weiter könnte die FMH folgende wichtige Aufgaben wahrnehmen: das Anbieten von Kommunikationsplattformen, das Analysieren von QM-Systemen und -Erfahrungen sowie deren Wirksamkeit, die Entwicklung von Benchmarkinginstrumenten sowie das Generieren und Vermitteln von Schlussfolgerungen. Zusätzlich, und basierend auf den Erfahrungen der Arbeitsgruppe Qualität der FMH, könnte ein Netzwerk von Personen aufgebaut werden, das sich auf QS/QM in der ambulanten Versorgung spezialisiert und dadurch den Mitgliedern den Zugang zu Qualitätsverbesserungen erleichtert.

Für ein solches Szenario müsste die FMH ihre Kompetenzen zusätzlich zu ihrem medizinischtechnischen Sachverstand in den Bereichen Evaluation und Analyse institutioneller und prozessorientierter Dimensionen der Qualität stärken. Von Vorteil wären auch ein intensiverer Austausch und ein verstärktes Networking mit Ärzteverbänden anderer Länder. Der Ansatz würde es der FMH erlauben, einen relativ neutralen Standpunkt in den Diskussionen zu QS/QM zu behalten, diesen jedoch durch selbstgewonnene Erfahrungen anzureichern. Mitglieder des Ärzteverbandes können individuell zu einzelnen Instrumenten der QS/des QM im ambulanten Bereich Stellung beziehen.

Die beschriebene Strategie würde es der FMH ermöglichen, ihre Erfahrungen und ihre Expertise zu erweitern und ihren Mitgliedern kompetente Beratung im QS/QM-Bereich zur Verfügung zu stellen. Ihre Rolle wäre auf diejenige einer Beobachterin und Mittlerin begrenzt. Die politische Debatte (unter anderem um das regulative Umfeld) würde so allerdings anderen Akteuren überlassen, die eine aktivere Rolle bei der Umsetzung entsprechender Ansätze spielen.

\section{Szenario 3 mit Fokus auf die Etablierung neuer Institutionen zu QS/QM}

Um eine aktivere Position in der gegenwärtigen und zukünftigen QS/QM-Debatte und dem regulativen Umfeld einzunehmen, müsste sich die FMH stärker in die Umsetzungsdiskussion einbringen und entsprechende Erfahrungen proaktiv entwickeln. Diesbezüglich sollte die FMH ihre eigenen Kompetenzen im QM-Bereich stärken, sie durch Partnerschaften bereitstellen oder durch Schaffung und Etablierung neuer Institutionen ermöglichen. Die Mitglieder der FMH müssten sich konstruktiv an der Ausgestaltung dieses Arbeitsfeldes beteiligen. Dadurch könnte der Ärzteverband unter Beweis stellen, dass traditionelle selbstregulative Mechanismen weiterhin ihre Berechtigung in einem sich wandelnden Umfeld haben, und gleichzeitig die führende Rolle in politischen Debatten ausbauen. Neben der historisch gewachsenen Rolle müsste die FMH ihre Vision erweitern und sich gegenüber unterschiedlichen Interessengruppen innerhalb des medizinischen Umfeldes im Hinblick auf einen interdisziplinären Ansatz der Gesundheitsversorgung öffnen, um diese dann in ein umfassendes Qualitätsverständnis einzubeziehen. Dazu ge-hören die verschiedene Berufskategorien (Ärzte, Pflegepersonal, Manager, Verwalter, Versicherer, Patienten und deren Vertreter usw.), die unterschiedlichen Anbieter von Gesundheitsdienstleistungen (ambulanter und stationärer Bereich, Langzeitpflege usw.) aber auch die diversen Akteure, die sich mit Qualitätsfragen befassen (Vereine/Stiftungen, kantonale Gesundheitsverwaltungen und Krankenkassen). Mit anderen Worten, die FMH müsste Koalitionen und Partnerschaften bilden, die das gemeinsame Verständnis und die Vorgehensweise innerhalb der QS/des QM fördern.

Ansatzpunkte für eine Kooperation könnte die Diskussion um ein gemeinsames Verständnis von Qualität im ambulanten Bereich sowie deren Erfassung und Messung sein. Mögliche Partner könnten Schweizer Akteure wie der Verein Outcome oder auch internationale Partner wie das Zentrum für Qualität in der Medizin (ÄZQ) oder die Kassenärztliche Bundesvereinigung in Berlin sein. Selbstevaluation und andere Instrumente der QS können benützt werden, um die Performance schrittweise zu verbessern. Mittels eines Benchmarkingsystems könnten Erfahrungen zur 


\section{Tabelle 1}

Mögliche Positionen der FMH zum Qualitätsmanagement in der ambulanten Versorgung.

\section{Szenario}

Fokus auf Qualitätssicherung:

Weiter- und Fortbildung, Curriculaentwicklung,

Förderung von wissenschaftlich validierten Abläufen

Fokus auf die Einführung von neuen Instrumenten zu QS/QM:

Einführung von laufenden Qualitätsverbesserungsmassnahmen wie zum Beispiel Qualitätszirkel, «peer audits», Selbstevaluationen oder Zertifizierungen

Fokus auf die Schaffung und Etablierung von neuen Institutionen zur QM:

Partnerschaft mit anderen Akteuren, die gemeinsame Kreation einer Institution im Bereich Qualitäts management oder die Entwicklung einer Qualitätssicherungs- bzw. -verbesserungsstrategie

\section{Chancen \\ Stärkung der Kernkompetenzen (derzeitiges Haupt- geschäft) sowie Garantenstellung für hohe medizi-} nische Dienstleistungen

Rolle als Moderatorin und gleichzeitig neutraler institutioneller Standpunkt zu Qualitätssicherung. Bereitstellung vertrauenswürdiger Information zur Entscheidungsfindung der Mitglieder der FMH

\section{Risiken}

Andere Akteure füllen Lücken in der Diskussion um institutionelle und Prozessdimensionen der Qualität. Berufsverbände können an Einfluss verlieren

Limitierter Einfluss auf das regulative und praktische Umfeld der Qualitätssicherung/QM

Proaktive Rolle zur Verbesserung von QS- und QMSystemen. Grosser Einfluss auf Änderungen und Einführung neuer Instrumente. Aktive Repräsentierung der Mitglieder in politischen Debatten

der Sichtbarkeit der FMH durch Partnerschaften. Vielzahl an neuen Aufgaben und limitierte Kapazitäten, diese aufzunehmen. Verlust der neutralen Position den Mitgliedern gegenüber. Konkurrenz zu Anbietern von QS/QM-Instrumenten
Qualitätsverbesserung gesammelt, ausgewertet und positive Erfahrungen und Beispiele den Mitglieder zugänglich gemacht werden.

In einem solchen Szenario wäre die FMH mit neuen Aufgaben konfrontiert, zu denen sie derzeit über wenig Know-how und Erfahrungen verfügt. Ein Engagement für Partnerschaften und Koalitionen birgt auch das Risiko des Verlusts der Sichtbarkeit gegenüber den Mitgliedern und der Öffentlichkeit sowie des Verzichts auf neutrale Positionen innerhalb der Diskussion um QS/QM. Dies würde die FMH möglicherweise von der Position einer reinen Interessenvertretung entfernen. Möglich wäre hier auch die Gründung einer Drittorganisation zusammen mit anderen Interessengruppen, die sich exklusiv mit dieser Thematik beschäftigt.

\section{Ausblick}

Die Diskussionen um die Verbesserung der QS und die Einführung von QM-Instrumenten im ambulanten Bereich stellen die FMH vor Herausforderungen. Die traditionelle Rolle selbstregulativer Mechanismen zur QS durch Standesorganisationen steht zur Debatte. Als historischer Garant der Qualität medizinischer Dienstleistungen wird sich die FMH der Frage stellen müssen, welche Funktionen und Aufgaben sie in Zukunft wahrnehmen möchte. Das 1996 in Kraft getretene Krankenversicherungsgesetz definiert Qualität nicht nur als das Resultat einer Behandlung und die Zufriedenheit der Patientinnen und Patienten, sondern auch als ein Mittel zur Kostendämpfung im Gesundheitssektor. Durch diese neuen Qualitätsstandards und das Auftauchen neuer QS/QM-Akteure im ambulanten Bereich muss sich die FMH neu positionieren, sofern sie in naher Zukunft eine Rolle bei Diskussionen und Entscheidungen zu Qualität medizinischer Dienstleistungen spielen möchte. Hierbei wird sich die FMH nicht nur auf die klassische Instrumente - die Lizenzierung von Ärzten sowie die Festlegung der Normen zur beruflichen Weiterund Fortbildung - beschränken können. Die verstärkte interdisziplinäre Zusammenarbeit verschiedener Berufskategorien, das Einbeziehen der Erwartungen von Patientinnen und Patienten sowie die wachsende Transparenz bei der Messung von Qualität und bei der Identifikation von Verbesserungspotentialen ist notwendig.

Bei der Ausgestaltung moderner Qualitätssicherungsmechanismen im Kontext umfassender QM-Systeme wird die klassische ärztliche Selbstregulation möglicherweise an Bedeutung verlieren und die Zusammenarbeit mit anderen Akteuren in den Vordergrund treten. Lobbying auf der Ebene von Entscheidungsträgern sowie das Fördern der QS/des QM bei den Mitgliedern der FMH werden ebenfalls wichtige Aufgaben der Standesorganisation sein, insbesondere da dies von grosser Bedeutung für die Finanzierung der Qualitätssicherungsmassnahmen sein kann.

Das immer komplexer werdende Umfeld im Gesundheitssektor, steigende Qualitätsanforderungen und zunehmender Kostendruck stellen die Ausarbeitung und Ausgestaltung umfassender Qualitätsstandards sowie deren Messung und Evaluation in den Vordergrund. Die FMH als traditionelle Akteurin im Bereich ärztlicher Qualitätssicherung sollte im Sinne einer kontinuierlichen Qualitätsverbesserung im Gesundheitswesen ihre langjährigen Erfahrungen in den Veränderungsprozess einbringen und dabei eine proaktive Rolle wahrnehmen. 